\title{
A novel carbon nanotubefet based bulk built-in current sensor for single event upset detection
}

\author{
T R RAJALAKSHMI ${ }^{1, *}$ and R SUDHAKAR ${ }^{2} \mathbb{D}$ \\ ${ }^{1}$ PSV College of Engineering and Technology, Krishnagiri, Tamilnadu 635 108, India \\ ${ }^{2}$ Dr. Mahalingam College of Engineering and Technology, Udumalai Road, Pollachi, Tamilnadu 642 003, India \\ e-mail: rajeereno@gmail.com
}

MS received 12 May 2015; revised 26 October 2015; accepted 15 January 2016

\begin{abstract}
The continuous scaling down of circuits has resulted in the development of carbon nanotubes (CNT) which provides a better alternative of silicon. High device packing densities is one of the advantageous factors of CNTFET compared to CMOS technology. This paper describes the new bulk current based built-in current sensor (BBICS) for the detection of single event upset (SEU) in CNTFET SRAM with less number of transistors compared to previous designs. The advantage of it is that its ability to detect low ranges of microcurrent. The complete circuit, both SRAM and Sensor are built with CNTFET. This also possesses the advantage of being used in the detection of negative current pulses with the addition of an inverter circuit. This circuit operates best for two different technology nodes. This built-in current sensor is connected to the bulk terminal of the CNTFET SRAM. PVT analysis and power dissipation analysis were done for the proposed circuit.
\end{abstract}

Keywords. CNTFET; bulk built-in current sensor; single event upset; SRAM.

\section{Introduction}

Carbon nano tube FET uses carbon nano tubes as channel instead of bulk silicon. They have the advantage of good electrical and optical properties and also a promising technology in circuit design. They also have the advantage of low power dissipation. Also their characteristics can be controlled by diameter. The choice of choosing CNTFET SRAM is it is faster and having good noise margin over CMOS SRAM. They seem to be efficient in Nanoscale integration. Single event upsets are the radiation effect which causes flip in the SRAM cell. These effects are due to ionizing particles in space or by the unstable ions in the packaging materials.

In this paper a novel built-in current sensor has been proposed which detects the SEUs at the time of occurrence. The earlier methods which used error control circuitries (ECC) lacked in certain performance factors which included power dissipation and area overhead. Also ECC detected the errors after the occurrence of faults and not at the instance of occurrence. Previously detection circuitries were connected to the power supplies. In this work we choose the detection of current from the bulk at the occurrence of SEU. The advantage is that the circuitry is

*For correspondence fast and without affecting the power supplies. The advantage of connecting the BICS in bulk rather than in power supplies is that the internal signals are not detected and only upsets are detected. And also from the previous researches bulk detection gives improved performance and low power dissipation [1]. In this paper Bulk BICS refers to the detection of bulk current with BICS.

CMOS inverter circuitry is used to amplify and invert the output which we get from the current to voltage converter. The inverter together with current voltage converter makes the sensor circuit. Optimized CNTFET inverter produces higher gain when compared to CMOS Inverters [2]. This property makes useful because our design makes use of CNTFET inverter.

The challenges behind CNTFET based Bulk current detecting BICS are

- Less number of transistors to be used to make the design a compact one.

- Have to detect nA current, since the SEU current flow is very low in CNTFET designs.

- Usage of CNTFET circuitry of varied CNTs for accurate results.

The paper is organized as follows. Section 2 deals with the previous works. Section 3 describes about bulk BICS approach. In section 4 experimental setup is elaborated. In section 5 simulation results have been discussed and section 6 deals with future research and conclusion 


\section{Previous works}

BICS proposed in Calin et al [3] and Vargas [4] is based on current monitoring. Vargas et al [4] proposed a new BICS scheme along with a latch which can detect current as high as $0.1 \mathrm{~A} \sim 280 \mathrm{pC}$ of collected charge. BICS proposed in [5] is based on voltage monitoring and it has less number of transistors compared to circuit in Calin et al [3]. It used current comparators and latch. In these designs sensing circuit was connected to power lines. The next set of works deals with bulk BICS. The value of the bulk current under normal conditions is low and during particle strike, it is very much high. (Wirth [6] deals with the bulk level detection at device level Bulk BICS in Neto et al [1] deals with bulk detection in combinational and sequential circuits. For memory circuit it has an inverter connection along with a NMOS for ground detection and a PMOS for VDD connection. Experiments in Zhang et al [5] deals with the development of Bulk BICS (BBICS) with less number of transistors and the reduced reset signals. This technique is able to detect for the bulk leakage current starting from $150 \mu \mathrm{A}$. Also this technique has an advantage of inbuilt latch. But in all the above, circuits detection was done in CMOS SRAM. In CNTFET SRAM where the very less amount of bulk current is detected, there is a necessity for a new BBICS.

The new design is aimed to solve the disadvantages in previous circuits. The proposed work has planned to have less area and to detect very minute bulk current variations on less than $1 \mu \mathrm{A}$ in CNTFET SRAM.

\section{Bulk BICS approach in CNTFET}

Bulk current based BICS (BBICS) possess the advantage of having high sensitivity when compared to other detections [1] The single event upset is modeled as a current pulse. Low LET heavy ions can be best described by the double exponential pulses [7] this modeling helps us to study about the various parameters affected by radiation. Current in the bulk region is usually at zero. The impact of current pulse makes the current in the bulk to increase and this abnormality is used in the detection.

The proposed circuit is connected to the bulk of CNTFET SRAM to read the SEU. This design works well even without latch. The developed circuit is used to detect SEU for n-bulk current as well as for p-bulk current. The advantage of this circuit is less complex with minimum number of transistors compared to the previous one and also the detection of nA current with CNTFET design.

Figure 1 is the general block diagram used for the detection of SEU in SRAM with bulk connection.

When compared to CMOS SRAM various factors have to be considered while the bulk terminal is intended to study CNTFET SRAM. First one is the chirality factor.

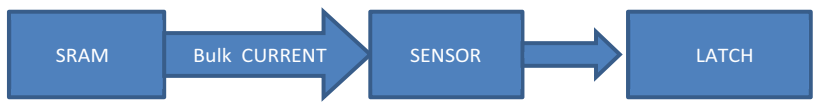

Figure 1. Block diagram.

Based on chirality factor, two parameters vary which include diameter and threshold voltage. When designing the circuit these also play a vital role. Variation in this chirality affects the output variation. Another factor to be considered is the number of carbon nanotubes used.

\section{Experimental setup}

The nanotube is placed between source and drain of the transistor and the nanotube plays the role of the channel. We have been using STANFORD CNTFET model. In the model (MOSFET like CNTFET) channel region is undoped while other regions of CNT's are heavily doped.

- Supply voltage VDD $=0.9 \mathrm{~V}$

- Chirality of tube $(m, n)=(13,0)$ and $(19,0)$ (zigzag)

- CNT PITCH = $20 \mathrm{~nm}$

- Physical channel length $(\mathrm{Lg})=32 \mathrm{~nm}, 22 \mathrm{~nm}, 10 \mathrm{~nm}$

- Mean free path intrinsic CNT $=200 \mathrm{~nm}$

- Mean free path doped CNT(Lss, Ldd) $=32 \mathrm{~nm}$

- Conventional CNTFET model is used. MOSFET like CNTFET operation is considered and SB-CNTFET effects not considered.

The ports definitions Drain, Gate, Source, Sub for the CNTFET are the same as that for a CMOS device. Here the current measurements are taken from the substrate terminal which can also act as a back gate in some applications. The study of internal structure of CNTFET with inclusion of capacitances is done from [8].

The physics of CNTFET model is referred from $[9,10]$.

The choice of MOSFET like CNTFET model is due to the fact that it is faster and suppresses the ambipolar conduction.

\subsection{BICS}

Various BBICS used with CMOS SRAM is studied and experimented with CNTFET SRAM. With them the minimum current detected is $150 \mu \mathrm{A}$. The proposed sensing circuit is able to detect even for minimum current of $90 \mathrm{nA}$ leakage in CNTFET technology. Since the bulk current was very low, current amplification along with sensing was tried. The current amplifier proposed by Wang et al [11] was tried for current amplification of bulk region, but failed for a short narrow pulse in CNTFET SRAM. The current mirror technique was used in some of the previously available BBICS Gill et al [12] which was tested in CMOS 

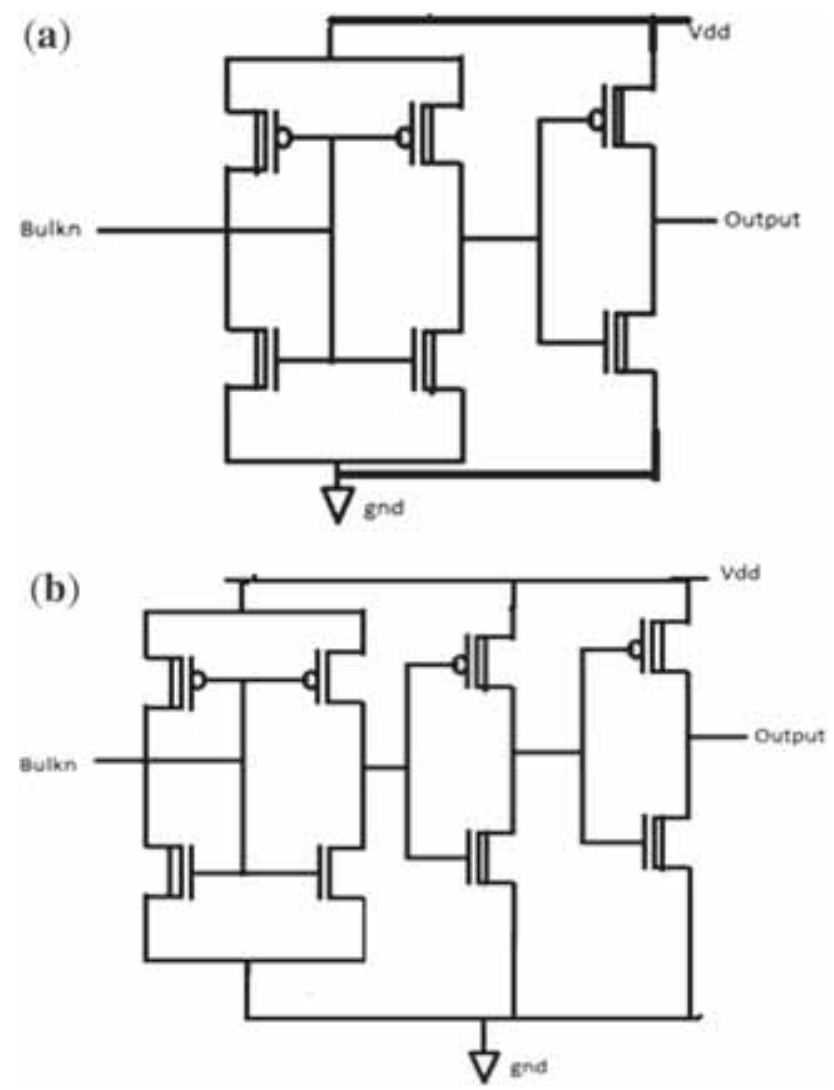

Figure 2. (a) BBICS sensor circuit. (b) BICS sensor for negative pulse detection.

SRAM. So several modified current mirrors from Baghtash et al [13] have also been tried for CNTFET SRAM based BBICS.

The current to voltage converter from Gupta et al [14] is modified to work as sensor. The current to voltage converter is used to give the output of the voltage equivalent of the current variation in the bulk terminal. That current to voltage converter is modified with additional circuitry and made to work as sensor. The output of the converter is given to the input of the CNTFET inverter for the desired output. When another inverter connected to the output of the inverter the circuit is able to detect negative pulses. The addition of inverter increases the amplitude and gain the output [15]. Figure $2 b$ gives the sensor circuit for the detection of negative pulse.

The built-in current sensor circuitry includes two PMOS transistors and two NMOS transistors along with the inverter. In this circuit logarithmic feature of the MOS transistors is used to get the equivalent voltage value of the varied current (here the change in the bulk current due to the introduction of SEU). The output value is an inverted output. The output value is given to the inverter for getting amplified and inverted proper error signal. First the sensor circuit is designed and various current pulses with different amplitudes were given for measuring the sensitivity of the newly designed sensor. The sensitivity of the newly developed sensor ranges from $90 \mathrm{nA}$ to $100 \mathrm{uA}$.

Figure 2a gives general BICS circuitry and figure $2 b$ BICS for negative pulses.

\subsection{BICS with CNTFET SRAM}

CNTFET SRAM affected by single event upset is considered for study. The SEU impacts on CNTFET SRAM cell is tested for read and write operations. Here rising time $t_{\mathrm{r}}=2.5 \mathrm{ps}$ is taken and $t_{\mathrm{f}}=5.5 \mathrm{ps}$ for ease of comparison with SRAM and the impact is analysed.

Figure 3a gives the circuit for BBICS connected to SEU injected SRAM.

STANDFORD CNTFET model is used for simulation. Simulations are executed for various channel lengths which include $32 \mathrm{~nm}, 22 \mathrm{~nm}$ and $10 \mathrm{~nm}$. Simulations are also executed for various chirality factors.

Figure $3 \mathrm{~b}$ the sensor circuit connected to SEU affected CNTFET SRAM (for PMOS sensing).

The experiment is initially carried out with $32 \mathrm{~nm}$ CMOS SRAM and then experimented for $32 \mathrm{~nm}, 22 \mathrm{~nm}$ and $10 \mathrm{~nm}$ CNTFET SRAM.

In most of the current mirrors and current sensors experimented with CMOS, processed the factor W/L ratio. But the advantage in CNTFET is that the ratio of PFET to NFET is 1:1 because both have almost the same current driving capability by having the same transistor geometry [16].

The same circuit is used to detect the bulk-p current of CNTFET SRAM design.

The leakage current in CNTFET measured in BULK region without the presence of SEU is almost close to Zero (10 E-13). And in the presence of SEU it is in the range of 2.5-8 $\mu \mathrm{A}$, and in some cases it is down to $\mathrm{nA}$ when the channel length is reduced. The BICS was developed with CMOS and verified with CMOS SRAM for detection in $32 \mathrm{~nm}$ technology and then extended for CNTFET SRAM.

CNTFET SRAM Design Specifications:

Number of transistors used: 14.

Technology: CNTFET (channel length $32 \mathrm{~nm}, 22 \mathrm{~nm}$ and $10 \mathrm{~nm}$ )

BICS: bulk current based current sensor

SRAM, BICS: CNTFET technology

Voltage Supply: $0.5 \mathrm{~V}$. For PVT variation $(0.3 \mathrm{~V}$ to $0.9 \mathrm{~V})$

Number of CNTs used in BICS: 2

\section{Simulation results}

Figure 4 gives the sensor output for positive current pulse and figure 5 for negative pulses.

Table 1 gives the detection possibilities for various set of bulk current ranges. 

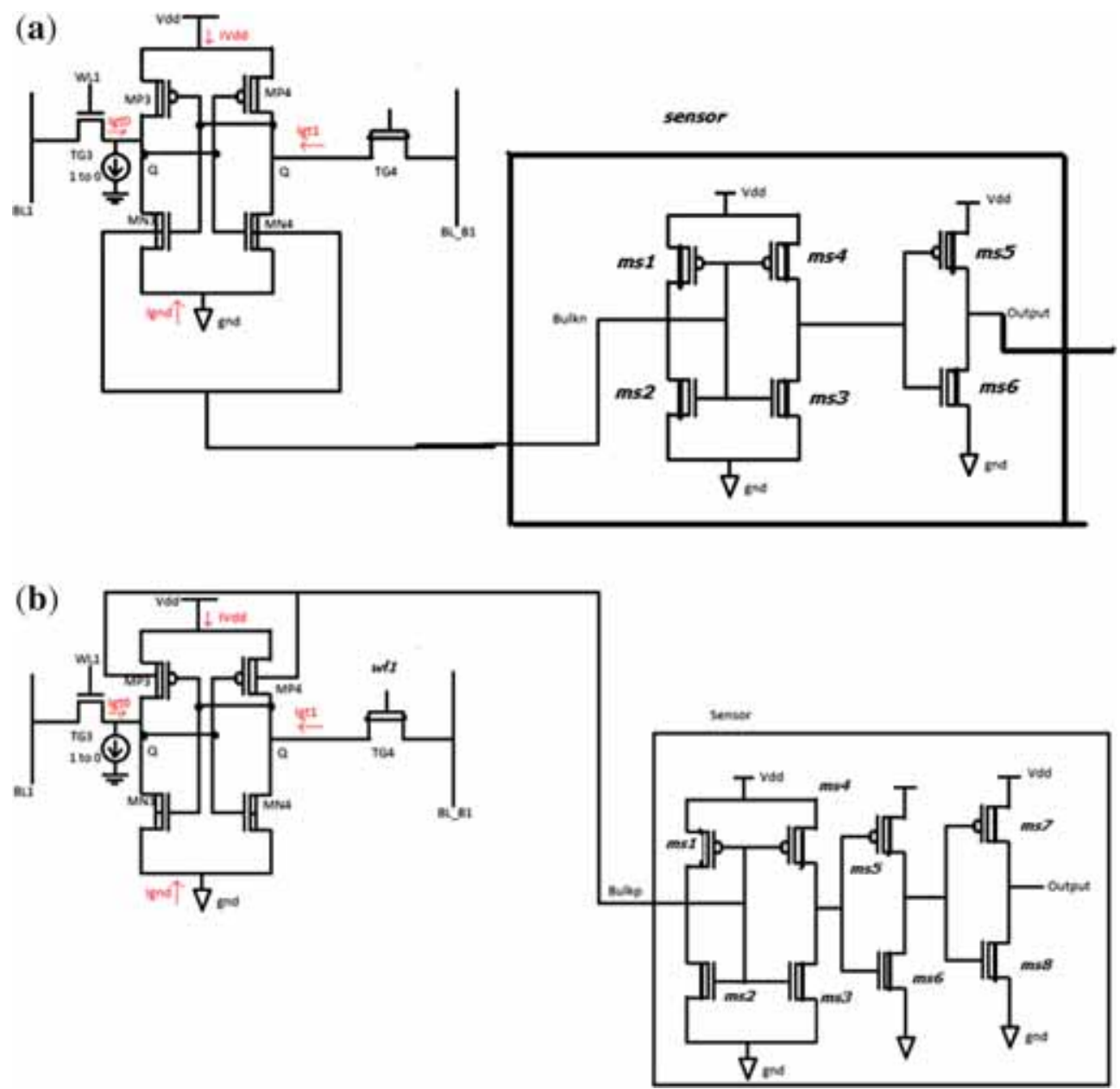

Figure 3. (a) SEU injected CNTFET SRAM with BBICS for N region. (b) BBICS connected to SRAM.
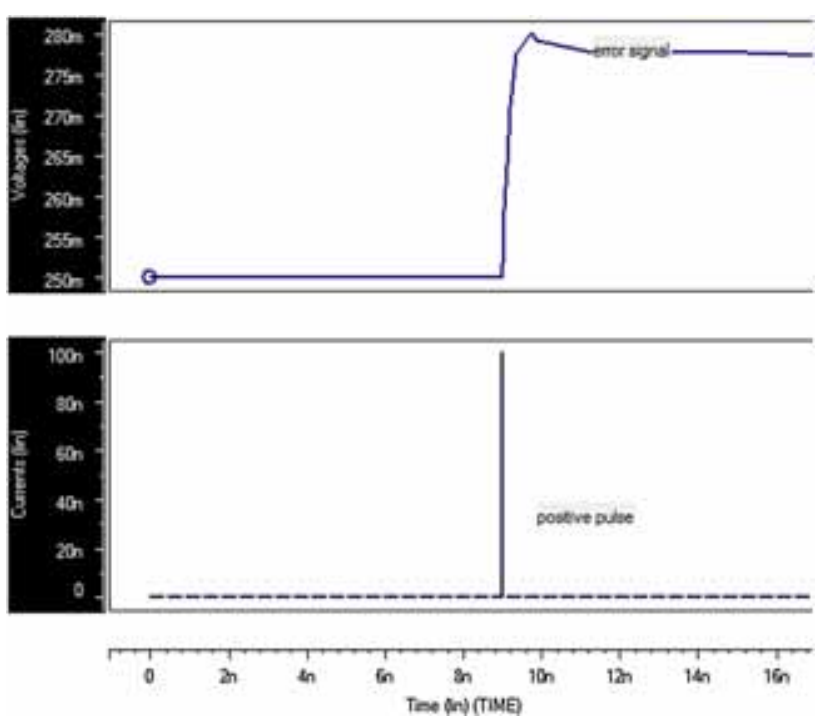

Figure 4. BICS detection for positive pulse.
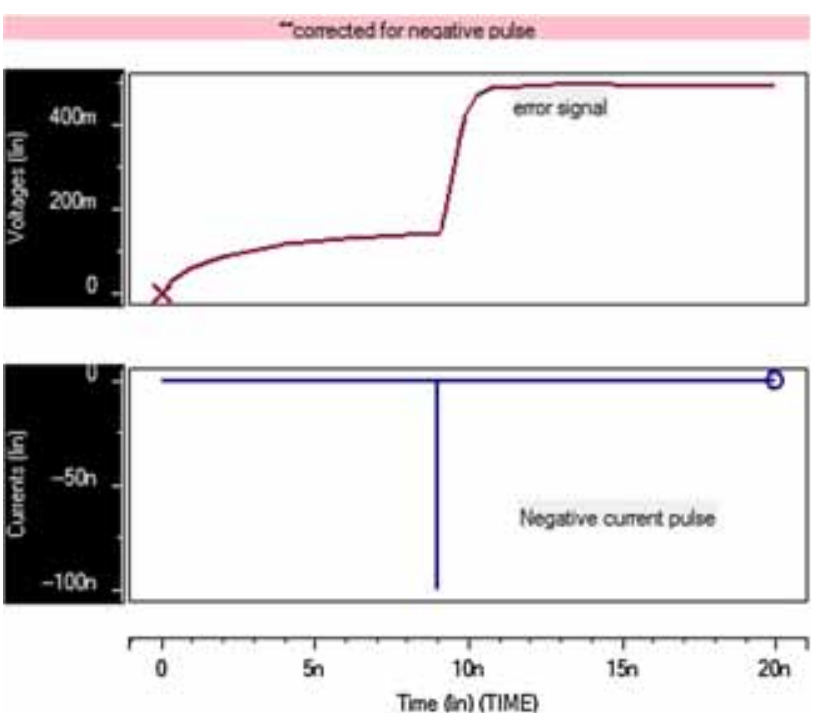

Figure 5. BBICS detection for negative current pulse. 
Table 1. Sensor detection for various current amplitudes.

\begin{tabular}{lccccc}
\hline Bulk current pulse value & $80 \mathrm{nA}$ & $90 \mathrm{nA}$ & $100 \mathrm{nA}$ & $1 \mu \mathrm{A}$ & $100 \mu \mathrm{A}$ \\
\hline Sensor output & $\mathrm{D}$ & $\mathrm{D}$ & $\mathrm{D}$ & $\mathrm{D}$ & ND \\
\hline
\end{tabular}

D, detected; ND, not detected.

Table 2. Sensor flipping and detection for various current pulses with different fall times for 1 to $>0$ (BICS Connected with SRAM).

\begin{tabular}{lllll}
\hline & & \multicolumn{3}{c}{ Fall time $t$ in $(\mathrm{ps})$} \\
\cline { 2 - 5 } Amplitude of current pulse I amp $(\mu \mathrm{A})$ & 2.5 & 3.5 & 5.5 & 7.5 \\
\hline 2 & $\mathrm{NF}$ & $\mathrm{NF}$ & $\mathrm{NF}$ & $\mathrm{NF}$ \\
& $\mathrm{ND}$ & $\mathrm{ND}$ & $\mathrm{ND}$ & ND \\
9.1 & FD & FD & FD & FD \\
19 & FD & FD & FD & FD \\
50 & FD & FD & FD & FD \\
\hline
\end{tabular}

NF, no flip; ND, no detection; F, flip; D, detection.

Table 3. Comparison chart.

\begin{tabular}{lcccc}
\hline Type & Proposed BBICS & BICS [17] & BICS [18] & BICS [12] \\
\hline CNTFET technology & Yes & No & No & No \\
Bulk current value & $90 \mathrm{nA}-100 \mu \mathrm{A}$ & Ranges & $160-200 \mu \mathrm{A}$ & - \\
& range & $>100 \mu \mathrm{A}$ & & Power and ground lines \\
Detection scheme & Bulk & Bulk & Bulk & 6 (NMOS alone) \\
Number of transistors (both PMOS and NMOS) & 14 & 16 & Done & - \\
PVT analysis & Done & - & & \\
\hline
\end{tabular}

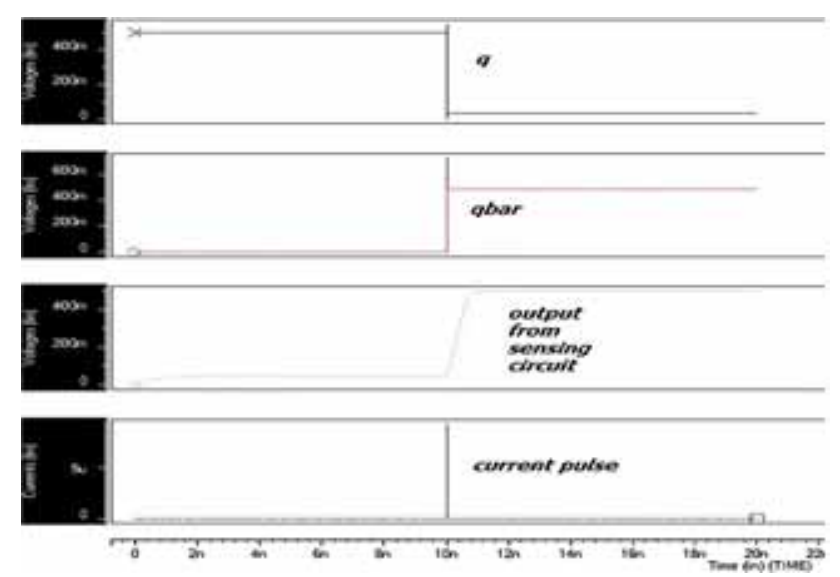

Figure 6. BBICS tested with SRAM for SEU detection in NMOS.

Table 2 gives the flipping and detection for various fall time values.

The sensor detects the output greater $90 \mathrm{nA}$ up to $100 \mathrm{uA}$ values. This sensor is used for the detection of SEU in CNTFET SRAM. The bulk current in CNTFET SRAM is very low when compared to CMOS SRAM, this sensor is found to be an effective one. The property of conversion of
$\mathrm{nA}$ current to equivalent voltage is considered. If the bulk current is very low, high gain is achieved due to increase in internal resistance [14]. And the bulk current value reaches some $\mathrm{mA}$ of current, variation gains reduce due to high voltage variation (table 2 ).

In SEU detection bulk current can drop as below $1 \mu \mathrm{A}$. The voltage gain will be low as it nears the bulk current and it drops to near 0 or VDD/2. Inverting amplifier is used to get error signal value as high. A gate with a regenerative property is used to get back the signal to a nominal voltage value. The output from the converter has the low value which is amplified by inverting amplifier. The converter and inverter together make the sensor circuit. The error signal goes high without latch. If latch is used reset signal is given to the latch through another NMOS transistor connected in series with transistor whose gate is connected the inverter output.

In second circuit where the converter is used for negative pulses two inverting amplifiers are required to get the necessary voltage gain in the output. Table 3 gives the comparative chart between various BICS.

The SEU detection in CNTFET SRAM is done for both READ and WRITE operations. During READ operation both BL and BLBAR were precharged to $\mathrm{VDD} / 2$. 

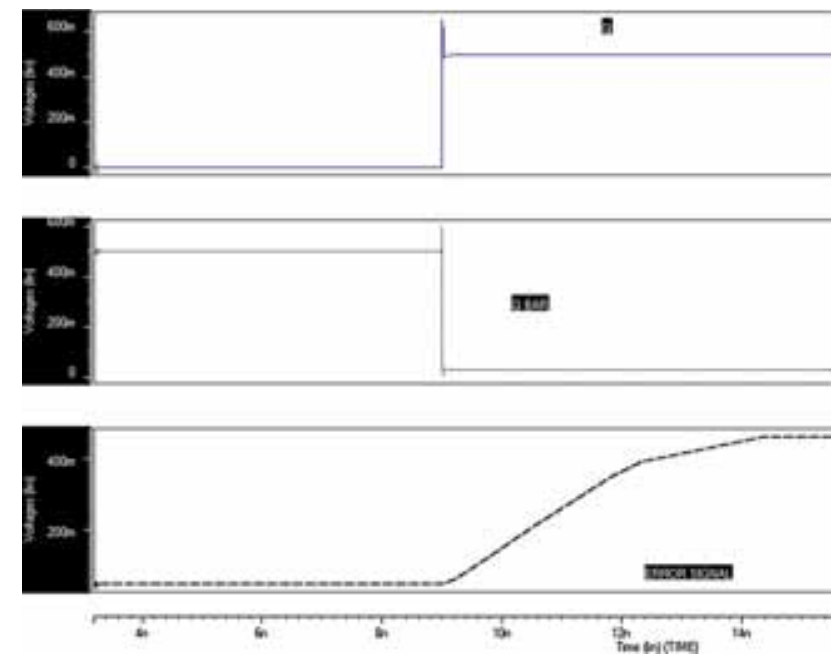

Figure 7. BBICS tested with SRAM for SEU detection in PMOS.
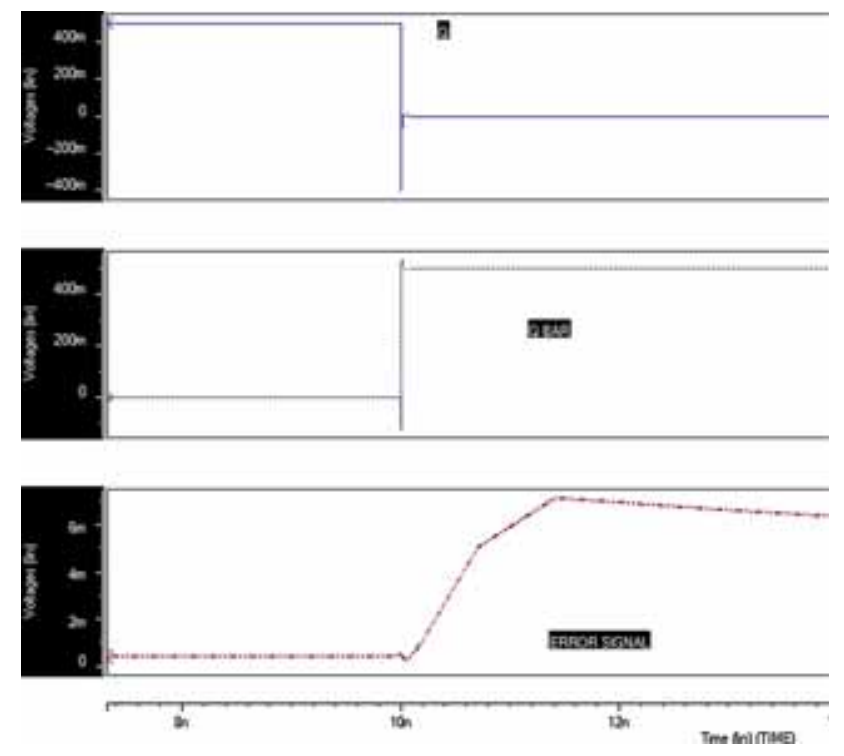

Figure 8. Flipping detection during read operation.

Figure 6 gives the flipping detection in CNTFET SRAM for $1 \rightarrow 0$ transition.

Figure 7 shows the flipping from 0 to $>1$ in PMOS affected CNTFET SRAM and the sensor detecting the flipping.

Figure 8 shows the flipping detection during read operation in NMOS.

\subsection{PVT variations}

The design is experimented for various chirality factors which include $m=13,15,17$ and the change in diameter values. CNTFETs are more sensitive to diameter values than width and length. Here we consider the diameter is varied evenly throughout the design. During analysis both sensor CNTFETs and SRAM CNTFETs were kept for the same diameter. The response of BBICS was good for less $m$ values than compared to higher values. The choice of CNTs also plays a vital role in the proper sensing. If the number of CNTs increased then it is equivalent to increasing the width of the CNTFET. This is because CNTs are the conducting channels in CNTFET between source and drain.

The temperature values are varied between $-10^{\circ}$ and $125^{\circ}$. For maximum temperature value the output falls down instead of maintaining the same value. Reducing the temperature does not affect the sensor output. Voltage is varied between $0.3 \mathrm{~V}$ and $0.9 \mathrm{~V}$. For minimum voltage point of $0.3 \mathrm{~V}$ flipping in the output is observed without detection in the sensor output. This is due to bulk current value being very minimal. For all the other voltage values flipping and detection were observed. And perfect error detection without delay is observed for $0.7 \mathrm{~V}$.

\subsection{Power dissipation analysis}

Total voltage source power dissipation measured with BBICS is $5.390397 \mathrm{e}-9$ watts and the total voltage source power dissipation measured without BBICS is $9.603 \mathrm{e}-12$ watts. Power dissipation on the introduction of BBICS is $5.3 \mathrm{nW}$, which is less compared to the previous techniques for typical process parameters.

\section{Future research}

Future research includes the enhancement of the circuit to increase its robustness by making to work under various temperature ranges. And the circuit works best for $32 \mathrm{~nm}$ and $22 \mathrm{~nm}$ technology. Hence BICS capable of detection in $10 \mathrm{~nm}$ range in an experimental level. This circuit is targeted with single SRAM circuit. Array of SRAM has to be connected for detection and efficiency has to be calculated. Enhancement of the sensing circuit for multiple bit upset detection has to be developed.

\section{Conclusion}

In this work a novel current sensor in CNTFET technology is discussed. It has the advantage of less area and has flexibility to work in different sizes. The efficiency and novelty of the circuit has been discussed. Power consumption is reduced. This work was experimented for two different technology nodes and for various diameter values. The sensor detection ranges up to $90 \mathrm{nA}$ which makes it useful for Low bulk current detections. The number of transistors used is also less whose value is seven for NMOS alone detection. And also for Bulk-p current detection the same circuitry can be used. 


\section{References}

[1] Egas Henes Neto, Ivandro Ribeiro, Michele Vieira, Gilson Wirth and Fernanda Lima Kastensmidt 2006 Using bulk built-in current sensors to detect soft errors. IEEE Micro 26(5): $10-18$

[2] Joerg Appenzeller, Richard Martel, Shalom J Wind and Phaedon Avouris 2003 IEEE Carbon nanotube electronics. In: Proceedings of the IEEE. 91(11), pp 1772-1784

[3] Calin Th, Vargas F L and Nicolaidis M 1995 Upset-tolerant CMOS SRAM using current monitoring: Prototype and test experiments. In: Proceedings of the IEEE international test conference, pp 45-53

[4] Vargas F, TIMA/INPG Lab., Grenoble, France and Nicolaidis M 1994 SEU-tolerant SRAM design based on current monitoring, digest of papers. In: Twenty-fourth international symposium on fault-tolerant computing, pp 106-115

[5] Zhichao Zhang, A nTao Wang, Li Chen and Jinsheng Yangew 2010 Bulk built-in current sensing circuit for singleevent transient detection. In: Twenty-third Canadian conference on electrical and computer engineering (CCECE) pp 1-4

[6] Gilson I Wirth 2008 Bulk built in current sensors for single event transient detection in deep-submicron technologies. Microelectron. Reliab. 48(5): 710-715

[7] Riaz Naseer et al 2007 Critical charge characterization for soft error rate modeling in $90 \mathrm{~nm}$ SRAM. In: Proceedings of the IEEE international symposium on circuits and systems, pp 1879-1882

[8] Razali Ismail, Mohammad Taghi Ahmadi and Sohail Anwar 2012 Advanced nanoelectronics, CRC Press

[9] Jie Deng and H.-S. Philip Wong 2007 A compact SPICE model for carbon-nanotube field-effect transistors including nonidealities and its application-Part II: Full device model and circuit performance benchmarking. IEEE Trans. Electron Dev. 54(12): 3195-3205
[10] Jie Deng and H-S Philip Wong 2007 A compact SPICE model for carbon-nanotube field-effect transistors including nonidealities and its application-Part I: Model of the intrinsic channel region. IEEE Trans. Electron Dev. 54(12): 3186-3194

[11] Wang C, Ahmad M O and Swamy M N S A 2002 CMOS current amplifier for very weak current operations. Solid-state circuits conference, 2002. ESSCIRC 2002. Proceedings of the Twenty-eighth European Publication Year, pp 751-754

[12] Balkaran Gill, Nicolaidis M, Wolff F, Papachristou C and Garverick S 2005 An efficient BICS design for SEUs detection and correction in semiconductor memories. In: Proceedings of the conference on design, automation and test in Europe, pp 592-597

[13] Faraji Baghtash H, Azhari S J and Monfaredi K H 2011 A novel very high performance CMOS current mirror with extremely low input and ultrahigh output resistance. Iranian J. Electr. Electron. Eng. 7(4): 235-240

[14] Kopal Gupta, Singh B P and Rockey Choudhary 2012 Design and analysis of current-to-voltage and voltage -to-current converters using $0.35 \mu \mathrm{m}$ technology. Int. J. Emerging Technol. Adv. Eng. 2(4): 457 (www.ijetae.com; ISSN 2250-2459)

[15] Lino M Samuel, K V Meena and Savita Y Patil 2013 CMOS current comparator with regenerative property. Int. J. Electron. Comput. Sci. Eng. 2(3): 992-999

[16] http://www.slideshare.net/RehanKunwar/cnfet-technolo gy?related $=1$

[17] Zhichao Zhang, Yi Ren, Li Chen and Nelson J Gaspard 2013 A bulk built-in voltage sensor to detect physical location of single-event transients. J. Electron. Testing 29(2): 249

[18] Alexandre Simionovski and Gilson Inacio Wirth 2012 A bulk built-in current sensor for SET detection with dynamic memory cell. In: IEEE Third Latin American symposium on circuits and systems (LASCAS), pp 1-4 